\title{
On stability and transport of holographic matter
}

\author{
Shu Lin*† \\ MPI, Munich \\ E-mail: slin@mpp.mpg.de
}

\begin{abstract}
We use gauge-gravity duality to study the stability of zero-temperature, finite baryon density states of $\mathscr{N}=4$ supersymmetric $S U\left(N_{c}\right)$ Yang-Mills theory coupled to a single massive $\mathscr{N}=2$ supersymmetric hypermultiplet in the large- $N_{c}$ and large-coupling limits. In particular, we study the Quasi-Normal Modes of the fluctuations of the bosonic fields, which gives the spectrum of the mesons. Despite circumstantial evidence that the system might be unstable, such as a finite entropy density at zero temperature and the existence of instabilities in similar theories, we find no evidence of any instabilities. We discover a pole on the imaginary frequency axis in a scalar meson two-point function, similar to the diffusion of spin at zero temperature in condensed matter physics.
\end{abstract}

Proceedings of the Corfu Summer Institute 2011 School and Workshops on Elementary Particle Physics and Gravity

September 4-18, 2011

Corfu, Greece

\footnotetext{
*Speaker.

${ }^{\dagger}$ Based on work with M. Ammon, J. Erdmenger, S. Müller, A. O’Bannon and J. Shock
} 


\section{The stability of a field theory at zero temperature and finite density}

Real world physical systems of interest often involve finite matter density. Examples include Quantum Chromodynamics (QCD) at finite baryon density and many condensed matter systems. The study of the thermodynamical and transport properties of these systems is in general difficult when the systems become strongly coupled. On one hand, the perturbative field theory method is not reliable. On the other hand, lattice simulation fails due to the sign problem at finite density and furthermore it cannot access physics involving real time. The gauge/gravity duality provides a solution to finite density field theory in the strongly coupled regime. In this work, we will study such a system using the gauge/gravity duality. The theory is $\mathscr{N}=4$ Super Yang-Mills theory plus $\mathscr{N}=2$ hypermultiplet. The theory has $S U\left(N_{c}\right)$ gauge symmetry and $U\left(N_{f}\right)$ global symmetry. The latter is analogous to the flavor group in QCD, and the diagonal $U(1)_{B}$ group of the flavor group gives rise to a baryonic charge. It is this baryonic charge that we give a density to. In particular, we will study the above field theory at zero temperature and finite baryon density. By virtue of the gauge/gravity duality, the field theory at strong coupling has a dual description in terms of $N_{c}$ D3 branes and $N_{f}$ D7 branes. A solution in the dual description has been obtained corresponding to a homogeneous state at zero temperature and finite density [1]. However, we expect it to be susceptible to multiple instabilities.

First of all, previous holographic study showed the system has a finite entropy at zero temperature [2]. While the D3 branes do not contribute to the entropy, the D7 branes have a finite contribution to the entropy, which cannot be reconciled with the third law of thermodynamics. A phase transition may happen before the zero temperature limit has been reached. Secondly, the fundamental multiplet contains both Dirac fermions and complex scalars. Due to the supersymmetry, the chemical potential we assign for the baryon number density is also an effective chemical potential for the complex scalar. It has been argued that complex scalar with large chemical potential and a quartic interaction is generically susceptible to Bose-Einstein condensation [1]. This argument does seem to include the system we will study as a special case. Thirdly, perturbative analysis on large $N_{c}$ QCD at asymptotic density has shown an instability, where the Fermi surface of the quarks are susceptible to formation of chiral density wave [3,4]. Our system shares many features with the large $N_{c}$ QCD at finite density, it is reasonable to expect the ground state at finite density may also be spatially modulated. Lastly, similar holographic studies on finite density system have indicated the presence of Chern-Simons (CS) term in the gravity theory can lead to ground state with spatial modulation [5] by violating the Breitenlohner-Freedman (BF) bound. Our system contains a Wess-Zumino (WZ) term in the D7 brane action, which acts like the CS term. Therefore, similar mechanism might also work in our system, making the homogeneous state in [1] unstable.

The goal of this work is to perform a systematic analysis of the bosonic, baryonic charge neutral operators corresponding to the fluctuations of the D7 brane fields. By analogy with QCD, these operators can excite mesons. By analyzing the dispersion relations of the mesons, we find the system is actually stable and shows some peculiar transport properties, reminiscent of the spindiffusion in condensed matter physics. 


\section{The gravity dual of the cold strongly coupled matter}

In the limit of infinite number of colors and infinite 't Hooft coupling, the $\mathscr{N}=4$ adjoint matter has a simple description in terms of near horizon limit of D3 branes. Furthermore, we focus on the "quenched limit", where the number of flavor for the $\mathscr{N}=2$ fundamental matter is much smaller than the number of colors, the dynamics of the fundamental matter reduces to the dynamics of probe D7 branes in the background of D3 branes. The near horizon limit of the background has the following metric and a self-dual Ramond-Ramond (RR) five-form:

$$
\begin{aligned}
d s^{2} & =G_{\mu v} d x^{\mu} d x^{v}=\frac{r^{2}}{R^{2}}\left(-d t^{2}+d \vec{x}^{2}\right)+\frac{R^{2}}{r^{2}}\left(d r^{2}+r^{2} d \Omega_{5}^{2}\right), \\
F^{(5)} & =\frac{4}{R^{4}}\left(r^{3} d x^{0} \wedge d x^{1} \wedge d x^{2} \wedge d x^{3} \wedge d r\right)-4 R^{4} d \Omega_{5},
\end{aligned}
$$

where $r$ is the AdS radial coordinate, with the field theory living at the boundary $r \rightarrow \infty$. The D7 branes share the same spacetime coordinates as the D3 branes, with the additional coordinates wrapping the $S^{3}$ of $S^{5}$. We separate the $S^{3}$ part of $S^{5}$ explicitly as follows:

$$
d \Omega_{5}^{2}=d \theta^{2}+\sin ^{2} \theta d \phi^{2}+\cos ^{2} \theta d \Omega_{3}^{2} .
$$

The decomposition allows us to write a simple RR four-form potential $C^{(4)}$

$$
C^{(4)}=\frac{1}{R^{4}}\left(r^{4} d x^{0} \wedge d x^{1} \wedge d x^{2} \wedge d x^{3}+R^{4} \cos ^{4} \theta d \phi \wedge d \Omega_{3}\right) .
$$

The dynamics of D7 brane is governed by the Dirac-Born-Infeld action with a WZ term. We will consider a single $\mathrm{D} 7$ brane, for which the action is given by:

$$
S_{D 7}=-T_{D 7} \int d^{8} \xi \sqrt{-\operatorname{det}\left(g_{a b}+\left(2 \pi \alpha^{\prime}\right) F_{a b}\right)}+\frac{\left(2 \pi \alpha^{\prime}\right)^{2}}{2} T_{D 7} \int P\left[C^{(4)}\right] \wedge F \wedge F,
$$

$T_{D 7}=\frac{g_{s}^{-1} \alpha^{\prime-4}}{(2 \pi)^{7}}$ is the D7-brane tension, $\left(\xi^{1}, \ldots, \xi^{8}\right)$ are the worldvolume coordinates, $g_{a b}$ is the induced metric of the D7-brane, $F_{a b}=\partial_{a} A_{b}-\partial_{b} A_{a}$ is the field strength associated with the worldvolume $U(1)$ gauge field $A_{a}$, and $P\left[C^{(4)}\right]$ is the pullback of the RR four-form to the D7-brane worldvolume.

A finite baryon density solution has been obtained in [1]. The solution corresponds to a nontrivial embedding of the D7 brane and worldvolume gauge field. Denoting $\rho=r \cos \theta$ and $y=$ $r \sin \theta$, the solution reads

$$
\begin{aligned}
y(\rho) & =\frac{1}{6} c \mathscr{N}^{-1 / 3}\left(\frac{d^{2}}{\left(2 \pi \alpha^{\prime}\right)^{2}}-c^{2}\right)^{-1 / 3} B\left(\frac{\mathscr{N}^{2} \rho^{6}}{\mathscr{N}^{2} \rho^{6}+\frac{d^{2}}{\left(2 \pi \alpha^{\prime}\right)^{2}}-c^{2}} ; \frac{1}{6}, \frac{1}{3}\right), \\
A_{t}(\rho) & =\frac{1}{\left(2 \pi \alpha^{\prime}\right)} \frac{1}{\varepsilon} y(\rho),
\end{aligned}
$$

where $\mathscr{N}=T_{D 7} 2 \pi^{2}=\frac{\lambda N_{c}}{(2 \pi)^{4}}$, and the parameters $c$ and $d$ are fixed in terms of the baryon chemical potential $\mu$ and quark mass $M$.

$$
\begin{aligned}
& c=\gamma \mathscr{N}\left(2 \pi \alpha^{\prime}\right)^{3}\left(\mu^{2}-M^{2}\right) M, \\
& d=\gamma \mathscr{N}\left(2 \pi \alpha^{\prime}\right)^{4}\left(\mu^{2}-M^{2}\right) \mu .
\end{aligned}
$$

Note the solution is valid for $\varepsilon \equiv \frac{\mu}{M}<1$ only. 


\section{Meson spectrum of the theory}

To study the meson spectrum of the theory, we consider all possible bosonic fluctuations of the D7 brane fields. These include the fluctuation of the embedding: $y(\rho) \rightarrow y(\rho)+\left(2 \pi \alpha^{\prime}\right) \chi$, $\phi=\left(2 \pi \alpha^{\prime}\right) \varphi$ and the fluctuation of the worldvolume gauge field: $A_{b}=\delta_{b}^{t} A_{t}+\delta A_{b}$. The equations of motion for the fluctuations are obtained by plugging the fluctuations into the action of D7 brane (2.4) and variating the action up to quadratic order in the fluctuations. The presence of a non-trivial background gauge field couples the equations of motion for the fluctuations. Apart from the zero sound mode that has been studied in [2], we find the fluctuations organize themselves into the following three classes:

$$
\begin{aligned}
& \chi=\Phi(\rho) e^{i \omega t-i k x_{3} \mathscr{Y}^{m}}, \delta A_{t}=\varepsilon \chi, \delta A_{3}=-\frac{\omega}{k} \varepsilon \chi, \delta A_{i}=0 \\
& \chi=\delta A_{t}=\delta A_{3}=0, \delta A_{i}=\Phi^{ \pm}(\rho) e^{i \omega t-i k x_{3} \mathscr{Y}^{m, \pm}}, \\
& \chi=\delta A_{t}=0, \delta A_{3}=\Phi(\rho) e^{i \omega t-i k x_{3}} \mathscr{Y}^{m}, \delta A_{i}=\frac{-i k \eta^{x x}}{m(m+2) \eta^{S 3}} \Phi(\rho) e^{i \omega t-i k x_{3}} \nabla_{i} \mathscr{Y}^{m}
\end{aligned}
$$

where $\eta_{a b} \equiv g_{a b}+\left(2 \pi \alpha^{\prime}\right) F_{a b}$ and $\eta^{S 3}=\frac{r^{2}}{\rho^{2}}$. $\mathscr{Y}^{m}$ and $\mathscr{Y}^{m, \pm}$ are scalar and vector spherical harmonics on $S^{3}$ with an integer eigenvalue $m$. The three classes of fluctuations lead to three master equations for the fields $\Phi$ and $\Phi^{ \pm}$:

$$
\begin{aligned}
& \partial_{\rho}\left(\sqrt{-\eta} \eta^{\rho \rho} \eta^{x x} \partial_{\rho} \Phi\right)-\sqrt{-\eta}\left(\omega^{2} \eta^{t t} \eta^{x x}+k^{2}\left(\eta^{x x}\right)^{2}+m(m+2) \eta^{S 3} \eta^{x x}\right) \Phi=0 \\
& \partial_{\rho}\left(\sqrt{-\eta} \eta^{S 3} \eta^{\rho \rho} \partial_{\rho} \Phi^{ \pm}\right)-\left(\sqrt{-\eta}\left(\eta^{S 3}\left(\omega^{2} \eta^{t t}+k^{2} \eta^{x x}\right)+\left(\eta^{S 3}\right)^{2}(m+1)^{2}\right)\right. \\
& \left. \pm 4\left(\rho^{2}+y^{2}\right)\left(\rho+y y^{\prime}\right)(m+1) \sqrt{\tilde{g}}\right) \Phi^{ \pm}=0 .
\end{aligned}
$$

To finish the discussion on the equations of motion, we further observe that the embedding field $\varphi$ and transverse components of the gauge field $\delta A_{1}$ and $\delta A_{2}$ satisfy the same equation of motion as $\Phi$. Therefore, we conclude that (3.2) describes all possible bosonic fluctuations apart from the known zero sound mode.

A quick look at the limit $\rho \rightarrow 0$ shows that the master equations for $\Phi$ and $\Phi^{ \pm}$are equivalent to the equation of motion for a massless scalar in $A d S_{2}$, therefore the BF bound is obviously not violated. To find any possible instabilities, we need to explore the full meson spectrum.

The meson states are given by the normalizable solution of the master equations (3.2). The condition of the normalizability gives the meson dispersion relation. This amount to find the QuasiNormal-Modes (QNM) of (3.2). An instability will be indicated by the QNM crossing the real axis from above in the complex frequency plane according to our choice of the Fourier transform. The common practice is to integrate the master equations from the horizon with an infalling boundary condition to the boundary, where it is demanded to be normalizable. However, the fact that the horizon is an essential singularity of the master equation (3.2) and the large imaginary part of the QNM makes this task numerically challenging. To overcome this difficulty, we use two different methods: a low frequency expansion [6] and a zig-zag method [7].

The low frequency expansion determines the analytic form of the retarded Green's function 


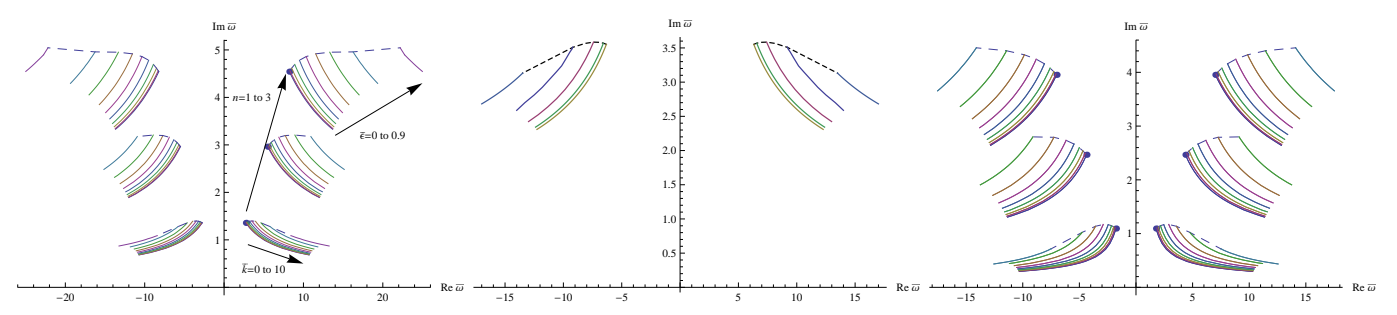

Figure 1: Left panel: The first three QNM of $\Phi$ for $m=0$, labeled by $n=1,2,3$. The solid lines describe the motion as a function of momentum $\bar{k} \in(0,10)$ and $\varepsilon \in(0,0.9)$. Middle Panel: The first QNM of $\Phi^{+}$ with $m=1$ for $\bar{k} \in(0,10)$ and $\varepsilon \in(0,0.8)$. Right panel: The first three QNM of $\Phi^{-}(\bar{\rho})$ with $m=1$ for $\bar{k} \in(0,10)$ and $\varepsilon \in(0,0.8)$.

for $\Phi$ and $\Phi^{ \pm}$to be:

$$
\begin{gathered}
G(\Omega)=\frac{b_{1}-i \Omega b_{0}}{a_{1}-i \Omega a_{0}}, \\
G^{ \pm}(\Omega)=\frac{b_{0}^{ \pm}-i \Omega b_{-1}^{ \pm}}{a_{0}^{ \pm}-i \Omega a_{-1}^{ \pm}},
\end{gathered}
$$

where $\Omega \equiv \bar{\omega} \sqrt{1-\varepsilon^{2}} . a_{i}, b_{i}, a_{i}^{ \pm}$and $b_{i}^{ \pm}$are functions of $\bar{k}, m$ and $\varepsilon$ that can be determined numerically without difficulty. Here $\bar{\omega}$ and $\bar{k}$ are dimensionless quantities scaled by the density. The QNM at low frequency, if exist, are given by $\Omega=-i \frac{a_{1}}{a_{0}}$ or $\Omega=-i \frac{a_{0}^{ \pm}}{a_{-1}^{ \pm}}$. An instability would correspond to $\frac{a_{1}}{a_{0}} \rightarrow 0^{-}$or $\frac{a_{0}^{ \pm}}{a_{-1}^{ \pm}} \rightarrow 0^{-}$. We find these do not occur for the lowest few integers $m$ and a wide range of $\bar{k}$ and $\varepsilon$, thus there are no unstable QNM in the low frequency regime. Interestingly, we find $\frac{a_{0}^{-}}{a_{-1}^{-}} \rightarrow 0^{+} \sim \bar{k}^{2}$ as $\bar{k} \rightarrow 0$. This gives rise to a diffusion mode $\Omega=i D(\varepsilon) \bar{k}^{2}$.

We also search for the QNM in the entire complex frequency plane using a zig-zag method. The method amounts to solving the master equations along a contour in the complex plane of the radial coordinate $\rho$, which bypasses the numerical issue near the essential singularity at the horizon. Detail of the method can be found in [7]. Applications of this method allows us to locate first few QNM for fields $\Phi$ and $\Phi^{-}$and the first QNM for $\Phi^{+}$as functions of $\bar{k}$ and $\varepsilon$ for the lowest integer $m$ in each case, corresponding to operators with the lowest dimension. Generically we expect an instability to occur for those most relevant operators. Fig.1 summarize our main results for the QNM for fields $\Phi, \Phi^{+}$and $\Phi^{-}$from left to right. We see none of the QNM cross the real axis from above, therefore no instability is found in our perturbative analysis of the fluctuations.

In additional to the three QNM we show in Fig.1 for the $\Phi^{-}$, we reproduce the diffusion mode found above using the low frequency expansion. Fig.2 shows the diffusive QNM of $\Phi^{-}$as a function of $\bar{k}$ and $\varepsilon$ for the lowest integer $m=1$. We also extract the diffusion constant $\frac{D(\varepsilon)}{\sqrt{1-\varepsilon^{2}}}$ defined by $\bar{\omega}=i \frac{D(\varepsilon)}{\sqrt{1-\varepsilon^{2}}} \bar{k}^{2}+O\left(\bar{k}^{3}\right)$ as a function of $\varepsilon$ for the lowest few values of $m$. The results are shown in Fig.3. The diffusion mode appears in the fluctuation of field $\Phi^{-}\left(\right.$or $\left.\delta A_{i}\right)$. For the lowest integer $m=1$, the dual operators are:

$$
\mathscr{O}^{I}=Q^{\alpha \dagger} \sigma_{\alpha \beta}^{I} Q^{\beta}
$$

where $Q^{\alpha}$ is the $S U(2)_{R}$ doublet of hypermultiplet scalars and $\sigma^{I}$ are $S U(2)_{R}$ Pauli matrices. The operators are charge neutral and form a triplet of the $S U(2)_{R}$. We will call this mode the "R- 


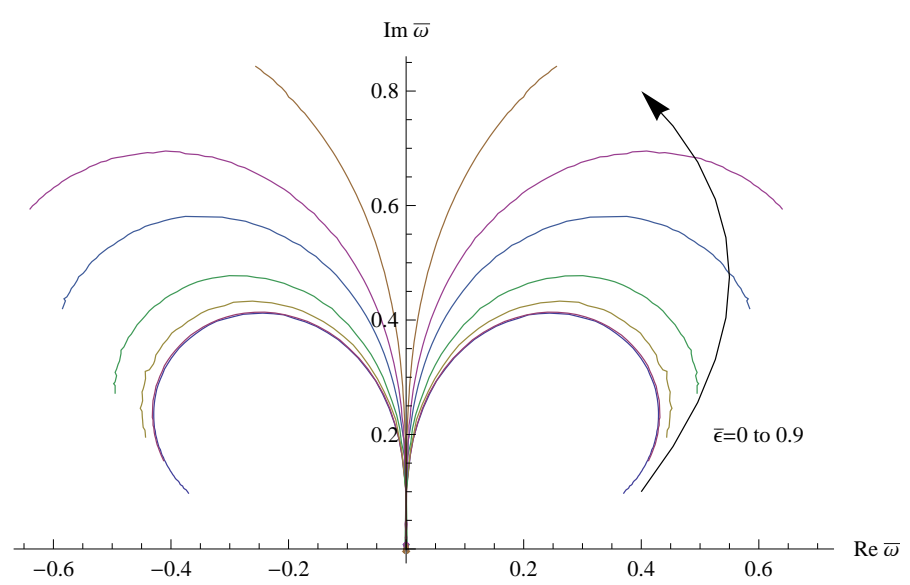

Figure 2: The diffusion mode of $\Phi^{-}$in the complex $\bar{\omega}$ plane, where each line indicates the motion, as a function of momentum $\bar{k} \in(0,9)$ for $m=1$. The different lines are for $\varepsilon=M / \mu=(0,0.1,0.3,0.5,0.7,0.8,0.9)$.

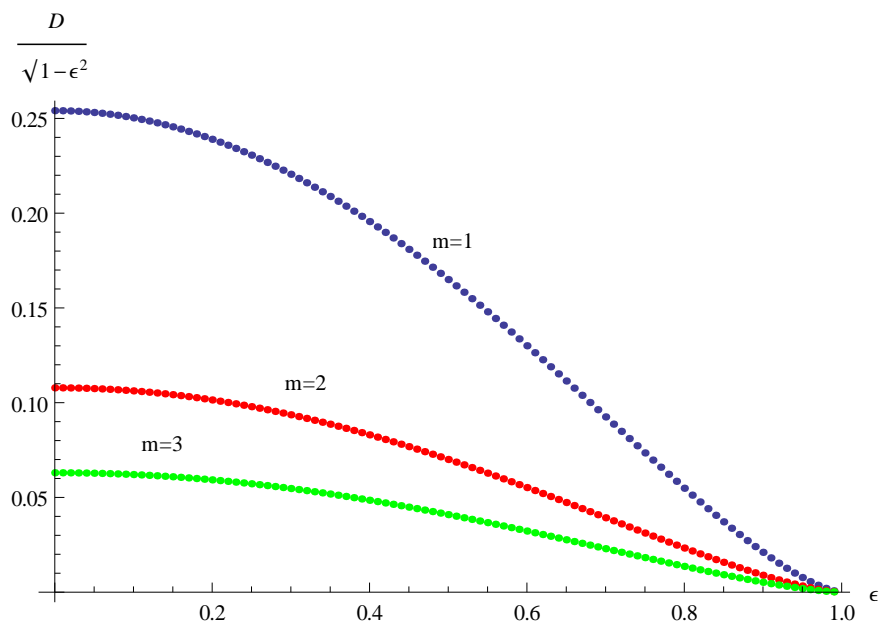

Figure 3: The "diffusion constant" $\frac{D(\varepsilon)}{\sqrt{1-\varepsilon^{2}}}$ for $m=1,2,3$ QNM versus $\varepsilon$.

spin" diffusion by analogy with conventional zero temperature spin diffusion in condensed matter physics.

\section{Summary}

We have studied the perturbative stability of $\mathscr{N}=4$ SYM theory coupled to a single flavor of $\mathscr{N}=2$ hypermultiplet at zero temperature and a finite baryon density. This is done by a systematic study of the fluctuations of the bosonic fields of the D7 brane. The QNM of the fields correspond to the dispersion relations of the mesonic operators in the dual field theory. We found that none of the QNM crosses the real frequency axis from above, indicating the absence of perturbative instability in our system. It is interesting to see if the conclusion remain trues for fluctuations for the fermionic fields and when non-perturbative effects are included. 
We have also found a diffusion mode corresponding to the fluctuation of $\delta A_{i}$. For the lowest integer $m$, the dual operators are charge neutral and form an "R-spin" triplet. We interpreted this mode as the diffusion of spin associated with $S U(2)_{R}$ symmetry. It remains an open question whether this diffusion mode is associated with the existence of a moduli space for the ground state [8].

\section{References}

[1] A. Karch and A. O'Bannon, JHEP 0711 (2007) 074 [arXiv:0709.0570 [hep-th]].

[2] A. Karch, D. T. Son and A. O. Starinets, arXiv:0806.3796 [hep-th].

[3] D. V. Deryagin, D. Y. .Grigoriev and V. A. Rubakov, Int. J. Mod. Phys. A 7 (1992) 659.

[4] E. Shuster and D. T. Son, Nucl. Phys. B 573 (2000) 434 [hep-ph/9905448].

[5] S. Nakamura, H. Ooguri and C. -S. Park, Phys. Rev. D 81 (2010) 044018 [arXiv:0911.0679 [hep-th]]. H. Ooguri and C. -S. Park, Phys. Rev. D 82 (2010) 126001 [arXiv:1007.3737 [hep-th]]. H. Ooguri and C. -S. Park, Phys. Rev. Lett. 106 (2011) 061601 [arXiv:1011.4144 [hep-th]].

[6] T. Faulkner, H. Liu, J. McGreevy and D. Vegh, Phys. Rev. D 83 (2011) 125002 [arXiv:0907.2694 [hep-th]].

[7] M. Ammon, J. Erdmenger, S. Lin, S. Muller, A. O’Bannon, J. P. Shock, J. Erdmenger and S. Lin et al., JHEP 1109 (2011) 030 [arXiv:1108.1798 [hep-th]].

[8] A. O’Bannon, Private communication. 